\title{
A morphogenetic analysis of facial growth
}

\author{
DONALD H. ENLOW, PH.D. \\ Ann Arbor, Mich.
}

'I'HIS study deals with a correlative morphologic and developmental analysis of the changes that take place during the postnatal growth of the face. It is based on information presented in previous studies concerned with growth and remodeling processes in the mandible and the nasomaxillary complex of the young human skull. ${ }^{1-3}$

Our present knowledge of postnatal facial growth is based largely on comparative phylogenetic correlations, ${ }^{3-7}$ anthropometric studies, ${ }^{8-10}$ and serial cephalometric roentgenography. ${ }^{11-17}$ These various studies have provided a great deal of quantitative information concerning relative growth movements, angular relationships, and rates of growth in the various facial planes. It is well known that the neonatal face is characteristically small in relation to the more precocious cranium. The face undergoes a progressive postnatal enlargement, however, and its various proportions become markedly changed with age. The over-all vertical and horizontal dimensions of the face come to bypass, in differential rates of growth, the slowing postnatal enlargement of the cranial base and the calvarium.

The purpose of the present report is to relate generalized patterns of facial growth with the corresponding patterns of structural remodeling which occur during the growth of the underlying facial bones. Continued increase in the size of the facial skcleton with age does not simply involve a generalized deposition of new bone on all outer surfaces. Rather, a complex series of remodeling changes takes place in all parts of the various facial bones as they increase in over-all size. These remodeling adjustments function in continuously maintaining the shape, the proportions, and the relationships of the various areas in each bone as the bone itself continues to grow. Detailed interpretations of these remodeling changes make possible an accurate analysis of the actual directions of growth in each individual region of each facial bone. It also makes possible an understanding of the mechanisms by which

From the Department of Anatomy, The University of Michigan. 


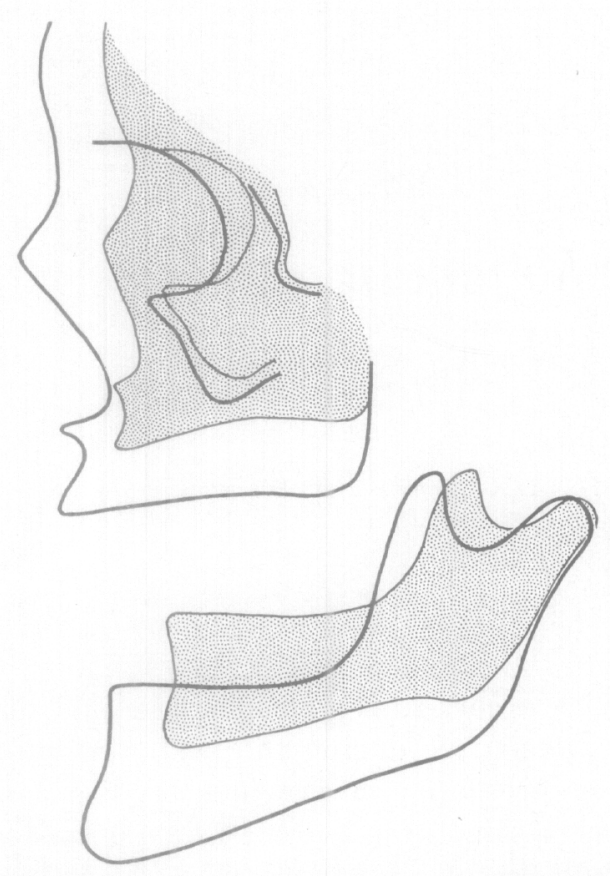

Fig. 1. The tracing overlay pictured here follows the standard method of registering (superimposing) "fixed" landmarks, including sella turcica and the anterior cranial floor. The time span represented is approximately 6 to 15 years of age. This cephalometric procedure enables the worker to visualize the apparent changes that take place during facial growth. These growth changes, which usually do not correspond with actual remodeling movements, are a consequence of the forward displacement of the mandible and the nasomaxillary complex. Note the "downward and forward"' manner of facial enlargement brought about by this process of forward thrust.

the localized parts of any given bone become successively relocated in new positions during progressive growth, as well as an interpretation of the structural changes which accompany such processes.

This study is based primarily on known morphogenic processes in the bone tissue itself, rather than on standard cephalometric methods. In the latter procedure, prominent radiographic landmarks that are considered to be relatively stable or "fixed" in position, such as the cranial floor and sella turcica, are selected as reference points. Tracings prepared from serial cephalograms are superimposed in such a way that these landmarks directly register or coincide. This method makes possible an evaluation of apparent growth movements (as in Fig. 1), and it permits accurate measurements of angular changes and relative distances moved during the growth of different bones or parts of bones. It is known, however, that actual directions of growth often do not correspond with such apparent growth movements, since a process of "forward thrust" is involved in both maxillary and mandibular growth. As some parts of these bones grow in predominantly posterior directions, they are continuously thrust or displaced in an opposite (anterior) 

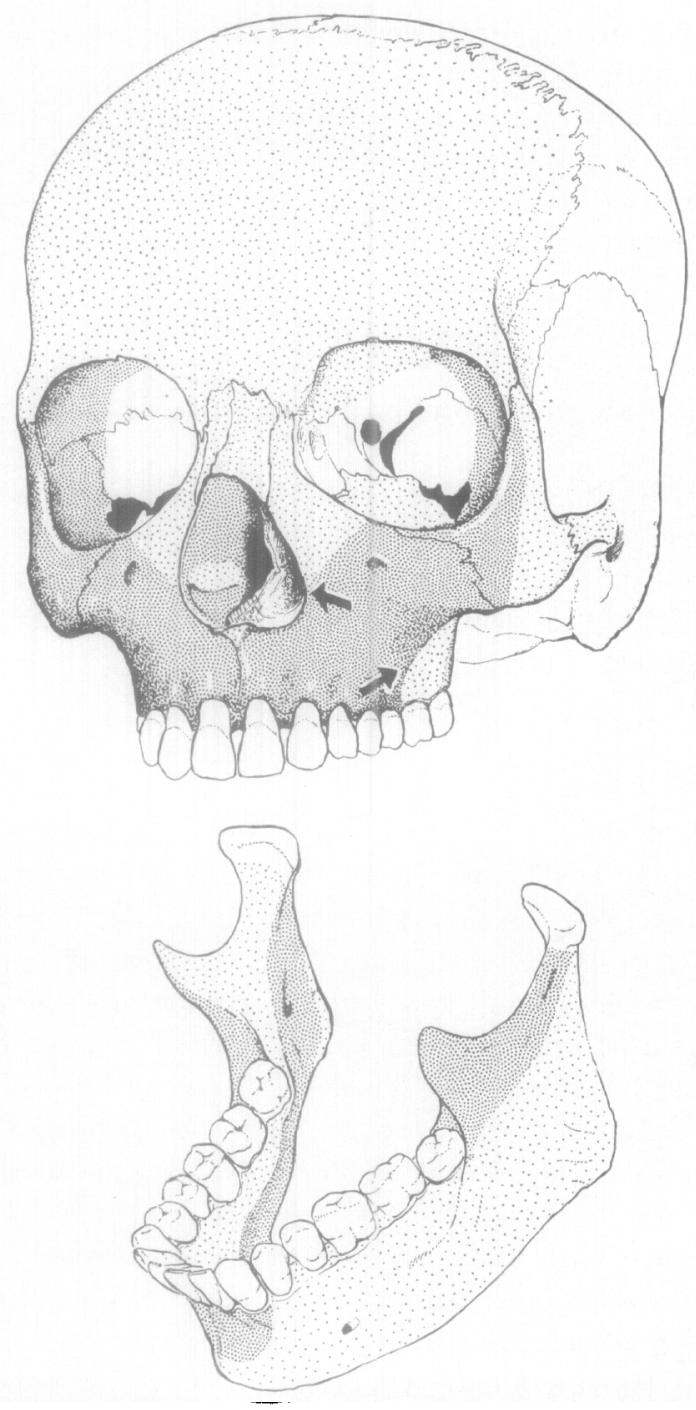

Fig. 2. Summary diagram showing the distribution of periosteal resorptive surfaces (dark stipple) and periosteal depository surfaces (light stipple). Note the growth reversals (indicated by arrows) between the mesial and distal (anterior and posterior) parts of the maxillary arch and between the nasal and premaxillary regions of the face. Remodeling changes involving these special regions are discussed in the text.

direction, thereby giving the over-all effect of an anterior mode of growth.

In Fig. 2 a summary diagram, based on my previous studies, ${ }^{2,3}$ shows the distribution of periosteal surfaces that undergo new bone deposition (light stipple) and those which undergo progressive periosteal resorption (dark stipple). These differential remodeling processes are involved in the over-all growth of the bones as they increase in size. Those periosteal surfaces that are depository in nature typically have a contralateral side which is re- 
sorptive. By this combined mechanism, the cortical plates involved move together in a progressively outward direction. Conversely, those outer (periosteal) surfaces that are resorptive usually have a corresponding endosteal side which is depository, thereby moving the cortex in an inward direction. These contrasting growth processes bring about composite remodeling movements that simultaneously proceed in a wide variety of individual directions in the different parts of the face, depending on orientation and the particular direction that each regional surface faces. For example, if a given surface is facing in a generally upward direction, resorption from that surface in eonjunction with new bone deposition on the opposite, downward-facing side of the cortex will bring about a shift of this entire cortical plate in a downward direction. Note, in Fig. 2, that the many local regions of the face are actually oriented in a complex variety of superior, inferior, posterior, anterior, lateral, and medial directions. Continued growth will, in general, maintain these same planes of orientation. The remodeling changes that accompany increases in size serve to adjust the shape and dimensions of each local area as it is constantly relocated into new positions.

\section{PROCEDURES}

Tracings were prepared from a series of sixteen serial cephalograms from normal Caucasian children, each series covering an age span of about 6 to 15 years. These tracings were then superimposed in such a manner that known surfaces of resorption and deposition and all directions of growth movement in each individual area correspond with the growth patterns illustrated in Fig. 2. The resulting overlay is pictured in Figs. 3 and 4, representing a composite of the most characteristic patterns observed. Landmarks, such as sella turcica, are not used as fixed registration points in the positioning of tracings.

Since it is known that the zygoma moves in a posterior course during growth and that the nasal areas of the maxilla grow forward, the tracings are registered so that both such movements are expressed. All other regions, including the premaxilla, the orbits, the maxillary body, etc., are likewise positioned and aligned so that their directions of growth all conform with the morphogenic pattern summarized in Fig. 2 . The purpose of this procedure is to provide a basis for demonstrating and evaluating the actual patterns of facial growth rather than the apparent directions of growth movement seen when standard cephalometric methods are used. The latter procedures, employing fixed reference points, are useful for determining and quantitating growth patterns produced as a consequence of forward displacement ("thrust"). They may lead to misunderstandings, however, in the sense that they can imply certain directions of growth in many areas where local growth actually proceeds in a quite different course. This situation is based on the fact that standard methods for superimposition of cephalometric tracings utilize reference points that are located on scparate and unrelated bones, each growing independently of the other and in entirely different directions and at different rates and times. Such techniques, however, are valid for their purpose, since the picture presented by these procedures provides a recon- 


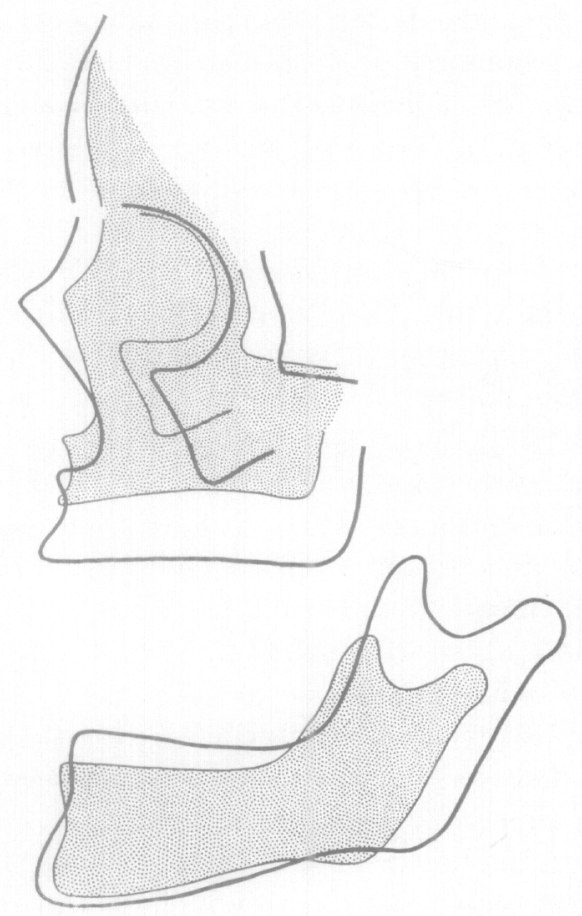

Fig. 3. Composite picture of superimposed cephalographic tracings showing regional patterns of facial growth most commonly observed in the present study. Significant variations, where present, are described in the text or in other figures. The age span represented is approximately 6 to 15 years of age. Tracings are registered in such a way that remodeling movements associated with the distribution of resorptive and depository surfaces (as in Fig. 2) are expressed. Fixed reference landmarks are not used. The patterns shown here represent actual growth movements, in contrast to the apparent growth changes pictured in Fig. 1. (See text for detailed descriptions.)

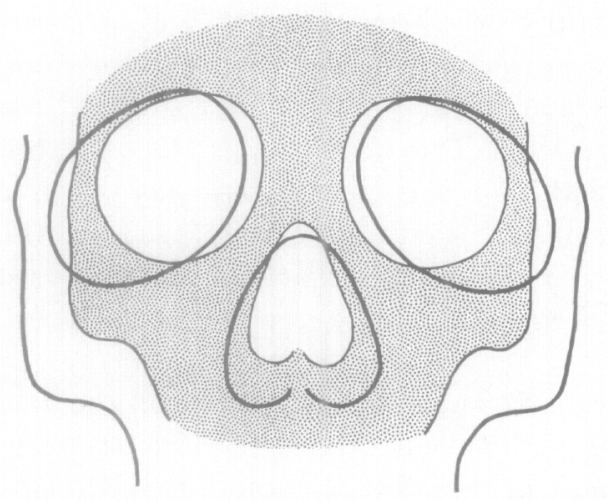

Fig. 4. Superimposed frontal cephalometric tracings. (See legend for Fig. 3.) 
struction of growth changes associated directly with forward thrust. The methods used in the present study serve to supplement this picture by providing an account of the remodeling changes that occur during facial growth uncomplicated by this secondary process of displacement.

When an age series of cephalometric tracings is superimposed and registered in such a way that all areas of surface deposition and resorption conform in a graduated series of stages, remodeling patterns related only to growth changes are revealed. It is important to realize that these growth movements represent the actual course and direction of development in each part of a given bone and that they cannot be visualized as such when considered in conjunction with the cranial base. It is only when the various regions of the craniofacial skeleton are evaluated separately in order to establish independent growth patterns and then combined as an interrelated composite that the over-all process of facial growth becomes meaningful.

When radiographic tracings are overlaid according to the procedure illustrated in Figs. 3 and 4, only a relatively slight degree of latitude is possible in the arrangement of the tracings. Thus, this method is not based on an arbitrary system of superimposition, even though actual fixed points are not utilized as reference marks. It is pointed out that this procedure is intended to demonstrate gross morphogenetic growth patterns and that although the distances involved in growth movements are close approximations, precise measurements should be interpreted with caution, since a small range of latitude is possible in the positioning of serial tracings.

My previous series of studies did not include the frontal bone. In order that the entire face can be considered as a whole, a detailed study of this bone has been made, using the same procedures outlined in past reports. The information gained from that study is included in the descriptions that follow.

\section{OBSERVATIONS}

THE FRONTAL REGION. The forehead grows in a generally anterior and slightly superior direction. This growth movement is the combined result of an expanding diploë and increasing thickness of the internal and external cortical laminae. The meningeal surface of the internal lamina is resorptive, as well as the endosteal side of the external lamina. The endosteal side of the inner lamina and the periosteal surface of the outer lamina are depository in nature. This combination produces a distinct drift of the forehead in an anterior (forward) direction. The presence in the prefrontal area of an inner meningeal surface that is resorptive is in contrast to the inner surface of adjacent coronal regions of the frontal bone, which are depository. It is possible that this resorptive meningeal surface may be associated with the disproportionately rapid growth of the prefrontal area in the cerebral frontal lobe of man.

The outer surface of the glabellar region of the frontal bone is entirely depository. Additions of bone on this surface contribute to the elevation and widening of the nasal bridge, in conjunction with movements of adjacent nasal and maxillary areas described in later paragraphs. 
In the supraorbital regions of the frontal bone in young human skulls with deciduous dentitions, the diploë located between the inner and outer laminae is noticeably more expanded than in other areas of the frontal bone. In older specimens with permanent dentitions, the diploë has become lacking altogether in the supraorbital region, in conjunction with the expansive enlargement of the paired frontal sinuses. These remodeling changes result in a marked anterior shift of the entire supraorbital and glabellar regions relative to the remainder of the frontal bone. This, in turn, produces a changing topographic contour of the frontal area in general, so that the bulbous forehead of the very young ehild becomes noticeably more sloping with inereasing age. The prominence of the supraorbital area in the older male, of course, is characteristically more pronounced. The actual anteroposterior distance between the forehead and the malar area in both sexes becomes inereased. This is a combined result of a forward-moving frontal bone and a posterior-moving zygomatic complex.

THE ORBIT. An interrelated combination of movements functions to enlarge the orbital cavities and to shift them in lateral directions. Fig. 4 shows that the approximate medial half of the roof and most of the floor of the orbital lining receive new deposits of bone on their outer (periosteal) surfaces. The entire medial (nasal) wall of the orbit is also depository in nature. Simultaneously, the lateral half of the roof and the entire lateral wall undergo resorption from the periosteal surface of bone lining the orbital eavity. Microscopic sections show a clear reversal near the vertex of the orbital roof between these endosteal and periosteal directions of growth (dark and light stipple). The lateral movement of the lateral orbital wall exceeds that of the medial orbital surface, thereby inereasing the horizontal dimensions of the entire eavity. This lateral movement occurs in conjunction with remodeling changes in the glabellar region of the frontal bone as well as the nasal part of the maxilla and the adjacent nasal bones. The result is an increased breadth and internal volume of the nasal bridge. Remodeling movements in the lateral orbital area proceed in combination with changes occurring in the zygomatic region, as described in a later paragraph.

The level of the inferior orbital rim drops somewhat as it becomes shifted in a lateral direction. This is due to the resorptive nature of the entire periosteal surface of the lateral orbital wall, including its basal part which is associated with the orbital process of the zygomatic bone. The lateral half of the roof of the orbital eavity becomes slightly elevated. This elevation is produecd by resorptive activity on the periostcal surface of the bony lining in this part of the orbit. This endosteal direction of growth undercuts the expanding supraorbital crest as the latter simultaneously grows in an anterior direction. Medial to the line of reversal between the depository medial half of the orbital roof and the resorptive lateral half, the cortical bone is composed of two layers. There is an inner zone of endosteal bone which was produced during earlier growth periods when this partieular area had a resorptive outer surface. As the entire orbit shifted laterally, the endosteal zone subsequently became covered with a new layer of periosteal bone, since the laterally 
moving medial part of the orbital roof successively occupied new areas that formerly were part of the lateral half of the roof.

In Fig. 4 we see that the lateral shift of the cutire orbit involves a process of "area relocation." In the floor of the orbit, the lateral part is lower in its level than the medial region. As the orbit shifts laterally, this medial area continuously comes to occupy regions that formerly were more lateral in their old position and were lower in level. The progressive deposition of new bone on the surface of the sloping orbital floor thus serves (1) to shift the entire orbit laterally and (2) to elevate the former lateral areas that have become relocated sequentially into the higher-positioned medial regions.

The entire lateral rim of the orbit also recedes in a progressive posterior direction. This is in conjunction with remodeling changes occurring in the zygomatic complex. As the rim moves in a backward course, however, the floor and the roof of the orbit are shifting in a slightly anterior direction. This shift is produced by the orientation of these regions. Since the floor is facing in a slightly forward direction, as well as laterally, additions of bone on this surface bring about growth in corresponding directions. The lining of the orbital roof faces away from this direction, so that the resorptive nature of this surface serves to shift the anterior part of the domed roof in an anterior direction as it simultaneously moves superiorly. These remodeling movements are linked to the changing relationships that occur between the forward-moving frontal and nasal areas and the posterior-moving zygoma.

THE MALAR REGION. In order to maintain its constant position relative to the face as a whole, the zygomatic complex grows in a progressive posterior direction as the dental areh becomes elongated by additions of new bone on the posterior margin of the maxillary body. The complex remodeling changes that bring about this posterior as well as lateral shift have been deseribed in detail in a previous study. ${ }^{3}$

The backward movement of the malar region, together with the forward growth of the supraorbital and nasal regions, serves to draw out the anteroposterior dimensions of the face. In Fig. 3 it is seen that the inferior rim of the orbit (anterior margin of the zygoma) and the forehead are in the same approximate vertical plane in the younger profile, with the zygoma perhaps extending somewhat beyond the frontal bone in some persons. In the older facial profile, however, the supraorbital region protrudes noticeably forward of the cheekbone and the lower margin of the orbital cavity. Note that the orbital ridges of the frontal bone extend forward of the eyeball in a mature face but that the eyeball extends forward of the inferior orbital rim.

The vertical dimensions of the zygomatic arch increase significantly in depth with age. This growth is primarily a result of bone deposition along the inferior border of the zygomatic bone and the zygomatic process of the temporal bone. The microscopic structure of these areas shows a massive accumulation of new bone deposits on these lower margins. Bone deposition also occurs along the superior border, but in much lesser amounts. The entire zygomatic arch, however, is apparently carried down by suture growth occur- 
ring between the zygomatic and frontal as well as the temporal and parietal bones, so that increases along the superior border of the arch are obscured by this downward shift. The superior border of the entire arch either remains in an approximately constant position or drops slightly. In none of the persons studied did the upper margin of the arch actually rise, even though this surface receives some periosteal deposits of new bone. Note the changing relationships that take place between the upper level of the zygomatic arch and the inferior border of the orbit as the latter continues to descend with incrcasing age.

THE NASAL REGION. The nasal portion of the maxilla and the nasal bones themselves are oriented in such a way that periosteal bone deposits carry this general region in a combined forward, lateral, and superior direction. In Fig. 3 it is seen that the anterior direction of nasal growth further draws out the depth of the face in conjunction with the receding malar region. This forward growth carries the tip of the nasal bone progressively farther forward than the downward-growing nasal spine. In the younger profile pictured in Fig. 3 the anteriormost point of the nasal bone lies on about the same vertical line as the nasal spine beneath it, in contrast to the expanded relationship seen in the older profile. These remodeling changes, combined with the unique growth mechanism of the premaxilla, produce the protruding bony nose, a singularly human facial characteristic.

A reversal in direction of growth occurs near the base of the nasal portion of the maxilla (Fig. 2, arrow) at that point where the nasal area grades into the premaxillary region. Below this point, the nasal wall of the maxillary bone is growing in a posterior (endosteal) direction, in contrast to the anterior (periosteal) direction of growth movement throughout the greater nasal area above the line. This growth pattern is concerned with the posterior and downward shift of the premaxilla, as discussed in a later paragraph. The combination of anterior growth along the upper part of the nasal area with recession from the lower region brings about an anterior and superior elongation of the entire nasal complex. The resorptive nature of the inferior part of the nasal wall serves to keep a constant relationship between the base of the nose and the receding premaxilla. This reversal also contributes to the markedly changing angulation of the anterior margin (free edge) of the nasal opening, as seen in Fig. 3 .

The periosteal surfaces of the nasal wall are oriented in such a manner that surface deposits of bone bring about growth in simultaneous anterior, lateral, and superior directions. As mentioned previously, the anterior and lateral growth serves to lengthen and broaden the nasal cavities. The superior direction of growth, however, does not bring about an actual elevation of the entire nose. Note that the tip of the nasal bones do not rise between the younger and older growth stages. Since the nasal contour is sloping, however, areas of the nose that were formerly lower (near the tip) become successively relocated into regions that are placed farther up on the elevated slope as the nose grows in length. Thus, the upward mode of growth functions to bring about this sequential process of superior relocation, even though the entire 
nose itself does not move in an actual upward direction. In most of the persons studied, the nasal tip itself was seen to drop in level (Fig. 3) as a result of continued anterior growth along the downward-facing anterior free margin of the nasal opening.

In several of the cephalographic series traced, the area of the nasal bridge was seen to have become slightly depressed with age (Fig. 5, e). In a previous study, ${ }^{3}$ it was found that this particular region represents an area of normal variation. The outer surfaces of the superior part of the nasal bones are resorptive in nature, in contrast to the more common depository surface, thereby bringing about a posterior and inferior shift of the nasal bridge. Since the distal half of the nasal bones is depository, however, a marked change in the angulation and profile of the entire nasal area is produced. In less extreme cases, it is possible that similar angular changes can occur simply by more rapid differential growth along the distal surfaces of the nasal bones than in the area of the bridge, thus producing comparable but less marked patterns.

In the following section, mechanisms involved in the extensive lengthening of the vertical dimensions of the nose will also be described. As a result of the increased height of the nose (produced primarily by the downward movement of the maxillary arch and hard palate), the nasal spine grows downward relative to the floor of the orbit as well as to the superior margin of the zygomatic arch (Fig. 3).

THE MAXILLARY ARCH. The elongation of the dental arch is a result of progressive bone deposition on the posterior surfaces of the maxillary body. As this occurs, the dental arch is also moving in an inferior direction by increased alveolar growth along the free margin of the entire arch. This inferior movement, however, does not account for the entire downward growth of the maxilla as a whole, since the inferior-moving nasal area (together with the palate) adds significantly to the total distance of this downward growth. Note the difference between total downward movement of the entire maxilla and the upward alveolar growth of the mandible as pictured in Fig. 3. It is also apparent that the maxillary arch of a very young child actually occupies the area of the future nasal eavity of the adult. The arch moves down by a process of area relocation, thereby increasing the vertical size of the nasal chambers. It can be seen that maxillary teeth must also be carried in an inferior direction, presumably by a process of downward drift in addition to the movement of eruption, much farther than the opposing mandibular teeth move in a superior direction during eruption.

As the maxillary dental arch moves downward, it is also increasing in lateral dimensions. In Fig. 2, a vertical line of reversal is seen (arrow) between that part of the maxilla that is anterior to the zygomatic process and the portion that is posterior to it. The posterior region increases in lateral dimensions by new bone deposition along the entire lateral periosteal surfaces of the maxillary body. Rostral to this line, however, the lateral as well as inferior growth of the maxillary arch proceeds by an entirely different remodeling sequence. The basis for these growth changes is schematized in 

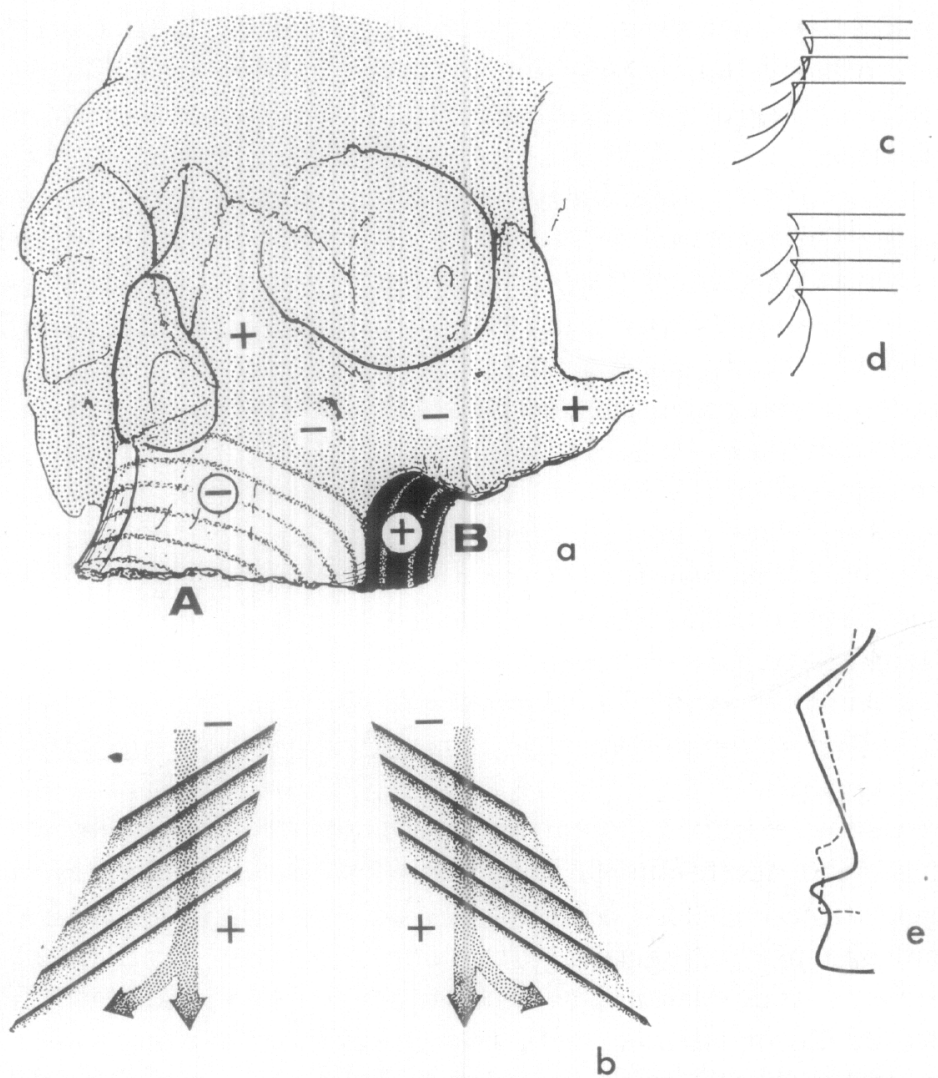

Fig. 5. a, Marked differences are present in the surface contours of the maxillary arch anterior to the zygomatic process $(A)$ and that part posterior to the zygomatic process $(B)$. Note that surface $A$ is oriented so that its outer (periosteal) side faces away from the downward direction of growth. This outer surface is resorptive (-). Surface $B$, in contrast, is oriented so that its outer side faces the direction of growth, which is downward as well as latcral. This surface is depository $(+)$. The line of transition between $A$ and $B$ oceurs along the ridge extending from the zygomatic process down onto the dental arch. As in area $A$, the anterior face of the malar region is also resorptive, a condition associated with the posterior drift of the zygoma. $b$, This figure shows schematically the downward movement of the entire anterior maxillary arch. The muzzle of the face (area $A$ in Fig. 5, $a$ ) grows downward by a combined process of periosteal resorption and endosteal deposition on the outer lamina of bone. The nasal and oral sides of the palate move in a corresponding direction. Note that the arch simultaneously becomes widened by an elongation of the free (alveolar) margin. Thus, the arch becomes enlarged even though its outer surface is resorptive. This is an example of the "V" prineiple described in previous reports.1-3 $c$ and $d$, Elongation of the alveolar margin can bring about a slight forward extension of the premaxilla, even though the periosteal surface of the area in general is resorptive. Note the "tilting" action associated with premaxillary remodeling, in which the upper part of this area undergoes regression while the lower part becomes extended in an anterior direction. In the sixteen cephalographic series studied, all but two showed a premaxillary region that appeared either to drop approximately straight down or to extend slightly forward (schematized in $c$ ). In two persons, however, the free alveolar margin apparently shifted in a posterior (distal) direction, as in $d$. $e$, In several of the persons studied, the bridge of the nose appeared to undergo progressive depression with age, in contrast to the more commonly observed pattern of elevation seen in Fig. 3. 
Fig. 5, $A$ and $B$, and involves the remodeling principle that "growth proceeds by a process of new bone deposition on those particular surfaces facing the actual direction of growth, together with proportionate resorption of bone from contralateral surfaces facing away from this direction." ${ }_{1-3}$ In Fig. 2 it is seen that the entire maxillary arch forward of the malar region has an outer periosteal surface that is resorptive in nature. Since the maxillary areh grows in a downward direction, it is apparent that the outer (periosteal) maxillary surface faces away from this direction of growth. As schematized in Fig. 5, $A$ and $B$, downward growth is accomplished by a combined process of resorptive removal from the outer surfaces of the anterior maxillary areh together with continued bone deposition along the entire opposite or lingual side. At the same time, the lateral dimensions become increased by an clongation of the alveolar area brought about by bone deposition along the alveolal' crests. Posterior to the reversal line on the maxillary area located beneath the zygoma, the orientation of the outer maxillary surface abruptly changes. The periosteal side of the posterior maxillary body faces in a generally downward direction (in contrast to the anterior part of the maxillary arch), so that new bone formation on this outer surface brings about corresponding downward as well as lateral growth.

In conjunction with the downward and lateral movement of the maxillary arch, the oral hard palate and floor of the nasal chamber simultaneously move in an inferior direction. This is accomplished by resorptive removal from the nasal side of the palate together with bone deposition on the oral side. The protruding nasal spine also moves in an inferior direction as a result of bone additions on its lower surface, with resorption from the superior side.

THE MANDiBLE. The mandibular condyle grows in a cephaloposterior direction through a process of endochondral bone formation. At the same time, the posterior border of the ramus grows in a backward course by progressive subperiosteal additions of new bone (Fig. 3). Because the condyle moves superiorly as well as posteriorly, the over-all distance traveled by the condyle exceeds that of the posterior-moving border of the ramus. Coincident with these growth movements, the coronoid process drifts in a corresponding posterior direction, although its backward movement is less than the posterior margin of the ramus. The result is a ramus that becomes continuously deeper in its anteroposterior dimensions. The ramus also increases significantly in vertical dimensions, thereby accommodating the marked downward growth of the nasomaxillary complex as well as the eruption of teeth. The complex remodeling adjustments involved in the specifie growth movements of the condyle, neck, notch, ramus, and coronoid process have been described in a previous study. ${ }^{2}$

The inferior border of the mandible just anterior to the gonial angle (antegonial notch) shows resorptive surface remodeling, since this notch successively occupies the regions formerly held by the posterior-moving, protruding gonial angle.

Alveolar bone growth along the superior border of the mandibular body brings about an increase in vertical dimensions. The amount of this growth in the mandible, however, is noticeably less than the opposing inferior direction 
of movement of the whole maxilla. The downward growth of the maxilla, as previously described, is a composite movement of alveolar growth in addition to nasal growth. As the dental arches of both the mandible and the maxilla grow toward each other, the relative positions of the two bones are continuously maintained by a proportionate vertical elongation of the mandibular ramus.

Except for the antegonial notch, bone is deposited along the entire inferior surface of the mandibular body, as shown in Fig. 3. Histologic examination of serial sections prepared throughout the length of the body shows an accumulation of compact periosteal bone deposits forming a massive outer cortex along the base and walls of the body, thereby providing increasingly stable support for the dental arch. Although the deposition of this bone results in a thick cortical plate, the actual distance moved in an inferior direction is less than the alveolar growth occurring on the superior side of the body.

The area of the chin receives thick deposits of periosteal bone, although much variation exists in the growth pattern of this particular region. ${ }^{2}$ The alveolar area just above the mental protuberance, however, is usually resorptive, and a degree of recession in a posterior direction occurs in this region. The structural result is the protruding chin, another singularly human facial characteristic. The significance of this remodeling circumstance is not fully understood, but it is noted that the corresponding incisor region of the maxilla is also resorptive, a factor associated with the downward growth of the maxillary arch. It is possible that the resorptive nature of the mandibular incisor region may represent an adjustment to the growth process in the maxillary bone and functions to stabilize ocelusion. Other related considerations have been discussed in previous reports dealing with the human mandible and maxilla., ${ }^{2,3}$

\section{DISCUSSION}

The concept that the face, in general, grows in a "forward and downward" manner is based on the standard cephalometric picture diagramed in Fig. 1. Many parts of the various facial bones do not, themselves, grow in such an anterior and inferior course. Growth actually proceeds in a complex variety of divergent, regional directions. The entire face, however, regardless of localized growth movements, becomes progressively repositioned or displaced in a forward manner, thereby obscuring actual growth patterns. $\mathrm{By}$ registering relatively fixed reference points, such as sella turcica, it is possible to demonstrate the manner of this anterior and inferior displacement of the various parts of the face relative to the cranial base. This particular method is of value since it permits the worker to visualize the topographic changes that take place in the gross appearance of the growing face. Because the effect of anterior thrust produces the visible morphologic relationships that one actually sees during the over-all growth of the child's face, methods utilizing selected landmarks in the cranial base provide meaningful information.

The purpose of the present study has been to provide an analysis of actual growth movements independent of this secondary process of forward thrust. Since virtually all parts of the growing bones in all regions of the face are independently undergoing localized growth and remodeling movements, there 
is no stable or fixed reference point that will enable the worker to superimpose precisely tracings of the progressive growth movements themselves. Sella turcica cannot be utilized for this particular purpose since remodeling processes in the maxilla are independent of this unrelated structure. Fixed points do not, of course, actually exist in any growing bone. Virtually all parts of all bones, including the elements of the craniofacial skeleton, undergo greater or lesser amounts of remodeling movements throughout active periods of body growth. Some selected postnatal regions or structures have been considered as relatively stable (such as the sella) and, as such, they are useful as a basis for assessing composite, cumulative growth movements between different bones in relation to each other. No such arbitrary point ean be utilized, however, in evaluating the actual remodeling changes that take place betwcen different bones or between different parts of the same bone. The procedures employed in the present study, rather than using fixed reference points, utilize the principle that all bony areas are, indeed, growing and moving and that such growth involves differential rates of increase and different directions of growth in the various parts of each individual bone. In order to establish the nature of such growth patterns, it is essential that cephalographic tracings of growth stages be positioned in such a manner that actual known directions of growth in each of the many parts of the various facial bones coincide. Each individual bone must be analyzed separately. Over-all patterns of facial growth may then be viewed by relating these individual patterns in each component bone, including the mandible, zygoma, zygomatic process of the temporal bone, maxilla, frontal, and nasal bones. This method is to be considered as a supplement to standard techniques involving the matehing of fixed reference landmarks. When both systems are utilized, the observer is able to visualize the actual as well as apparent modes of growth. It is suggested that known cephalometric values might be translated and related to the growth patterns revealed when tracings are superimposed according to the present procedure. Such studies are encouraged.

THE PREMaXILla. The succession of growth changes involved in the downward movement of the maxillary incisor region is schematized in Figs. 5, $A$ and $B$. This sequential remodeling process involves resorptive removal of bone from the outer, labial side of the premaxilla together with progressive and simultaneous deposition of bone on the lingual surface. Thus, the surface that faces the direction of growth receives new bone deposits, while the contralateral surface facing away from this direction, which is the outer side of the premaxilla, is resorptive.

As the free margin (alveolar crest) of the premaxilla moves down, the upper, more posteriorly situated part of the bone becomes progressively relocated in a backward direction into former areas that had previously protruded more anteriorly. The resorptive nature of the outer cortex, which functions in this downward growth as deseribed above, serves to bring about this sequential recession of the premaxilla in order to align continuously the moving outer contour of the entire premaxillary bone. Although the premaxilla is recessive in nature, as shown in Fig. 5, $\mathrm{C}$, actual recession can be limited to the remodeling 
adjustments just described. Because the crest of the alveolar region becomes lengthened by new bone deposition, the free margin of the premaxilla does not necessarily undergo an actual recession in a posterior direction. Rather, the elongation of the alveolar crest results in a maxillary margin that drops straight down or extends in a slightly anterior direction.

In two of the sixteen series of cephalograms studied, the circumstance pictured in Fig. 5, $D$ was observed. In this situation, not only the upper portion of the bone, but the terminal alveolar part as well, appeared to undergo posterior movement. A statistically large number of individual subjects should now be studied in order to determine the degree of actual spread and the incidence of occurrence of the remodeling differences shown in Fig. 5, $C$ and $D$. It would appear that this particular morphologic area may represent a region of normal developmental variability. It is suggested that extremes might contribute to variable degrees of proguathism or to an abnormally receded incisor region.

The "tilting" action seen in Fig. 5, $C$, in which the upper area undergoes backward movement while the lower region elongates in an anterior direction as a result of increased alveolar crest deposition, may bring about a change in the angulation of the entire premaxillary surface. It may also contribute to an excessively protruded incisor region if carried to extreme. Further, considering the variability found in the corresponding incisor region of the mandible, an extreme of the situation seen in Fig. $5, D$, might result in a variety of aberrant occlusal relationships between maxilla and mandible.

FACIAL TOPOGRAPHY. As the face matures with age, a number of characteristic, age-related morphologic changes occur. These are produced by remodeling alterations that take place in the facial skeleton during over-all growth. The major changes observed in facial features associated with growth are outlined below.

The bulbous appearance of the forehead and the upright angle of the entire face are reduced by expansion of the supraorbital ridges, protrusion of the glabella, elevation (or depression) of the nasal bridge, and elongation of the nose. Alveolar growth in mandibular and maxillary incisor regions also contributes to the changing angulation of the face as a whole.

The nose becomes longer and wider and increases noticeably in vertical size. The nasal bridge becomes progressively more prominent in many persons.

The orbital cavities move farther apart. The adjacent malar (cheek) area becomes much more massive because of an increased depth resulting from growth additions along the inferior border of the zygoma. The zygomatic arehes move laterally, away from each other, thereby increasing the breadth of the face.

The diminutive mandible of early youth is replaced by the squared lower jaw of the adult. This is a result of the massively growing trihedral eminences and the lateral gonial flares. Increasing permanent dentition, as well as the growth of maxillary and mandibular alveolar bone, produces the full external appearance of the entire masticatory region. The marked growth of the mandibular portion of the face can obscure the proportionate extent of growth taking place in the malar region. 
The chin becomes progressively more prominent. This factor also contributes to the changing facial angle and profile.

A more expanded curvature develops between the nose and the cheek as a result of their divergent directions of growth.

The entire face becomes longer in a vertical dimension as a composite result of mandibular ramus growth and downward growth of the nose and maxilla. The face as a whole is displaced downward in relation to the cranium. As a consequence, changing spatial relationships take place between the various parts of the face and such independent landmarks as sella turcica or the auditory meatus.

The face becomes noticeably deeper in a horizontal (anteroposterior) plane because of an interrelated combination of several remodeling changes, including forward elongation of the nose, anterior thrust of both upper and lower jaws, backward shift of the lateral orbital rim, posterior movement of the zygoma, and posterior extension of the dental arches. Since these various movements proceed in different directions, the entire face is, in effect, drawn out in several divergent directions.

\section{SUMMARY}

This study is an analysis of facial growth patterns based on known remodeling changes that occur in the various bones and parts of bones in the human face. Serial cephalometric tracings were used to correlate these remodeling changes with successive growth stages.

The results of the study provide an account of the actual changes that take place in the facial skeleton during craniofacial growth. The standard system of cephalometric superimposition using fixed reference landmarks provides, in contrast, a picture of apparent growth movements due to the forward thrust (displacement) of the maxilla and mandible away from the cranial base. A more meaningful and complete understanding of over-all facial growth is gained when both systems are used, one supplementing the other.

Each component area in the frontal and nasal bones, the maxilla, the zygoma, and the mandible are considered separately. Regional growth movements are described, and relationships are analyzed. A correlation is presented between characteristic age changes that take place in the topographic features of the face and the corresponding remodeling changes that accompany over-all facial growth.

This work was supported, in part, by United States Public Health Service Grant DE09103. The author is indebted to Dr. Robert Moyers of the Department of Orthodontics, University of Michigan, for making available the serial cephalograms used in this study. Dr. A. T. Storey of the Department of Orthodontics provided assistance in the interpretation of cephalograms. The cooperation of Dr. William Mills, Acting Direetor of the University School, in organizing and collecting histories of the subjects used is gratefully acknowledged. The series of illustrations used in this report was prepared by $\mathrm{Mr}$. W. L. Brudon.

1. Enlow, D. H.: Principles of Bone Remodeling, Springfield, Ill., 1963, Charles C Thomas, Publisher. 
2. Enlow, D. H., and Harris, D. B.: A Study of the Postnatal Growth of the Human Mandible, AM. J. ORThodontics 50: 25-50, 1964.

3. Enlow, D. H., and Bang, S.: Growth and Remodeling of the Human Maxilla, AM. J. ORTHODONTICS 51: 446-464, 1965.

4. Gregory, W. K.: Our Face From Fish to Man, New York, 1929, G. P. Putnam's Sons.

5. Krogman, W. M.: The Problems of Growth Changes in the Face and Skull of Anthropoids and Man, Dental Cosmos 72: 624-630, 1930.

6. Todd, T. W.: Prognathism; a Study in Development of the Tace, J. Am. Dent. A. 19: 2172-2184, 1932.

7. Björk, A.: Some Biological Aspects of Prognathism and Occlusion of the Teeth, Acta odont. scandinav. 9: 1-40, 1950.

8. Hellman, M.: The Face in Its Developmental Career, Dental Cosmos 77: 685-787, 1935.

9. Goldstein, M.: Changes in Dimensions and Form of the Face and Head With Age, Am. J. Phys. Anthropol. 22: 37-89, 1936.

10. Davenport, C. B.: Postnatal Development of the Head, Proc. Am. Phil. Soc. 83: 1-26, 1940.

11. Broadbent, B. H.: The Face of the Normal Child, Angle Orthodontist 7: 183-208, 1937.

12. Brodie, A. G.: On the Growth Pattern of the Human Head From the Third to the Eighth Year of Life, Am. J. Anat. 68: 209-262, 1941.

13. Brodie, A. G.: Facial Patterns: A Theme on Variation, Angle Orthodontist 16: 75-87, 1946.

14. Brodie, A. G.: Behavior of Normal and Abnormal Facial Patterns, Am. J. Orthodonties \& Oral Surg, 27: 633-647, 1941.

15. Brodie, A. G.: Late Growth Changes in the Human Face, Angle Orthodontist 23: 146$157,1953$.

16. Björk, A.: The Face in Profile, Svenska tandl. tidskr. 40: 56, 1947.

17. Lande, M. J.: Growth Behavior of the Human Bony Facial Profile as Revealed by Serial Cephalometric Roentgenology, Angle Orthodontist 22: 78-90, 1952. 